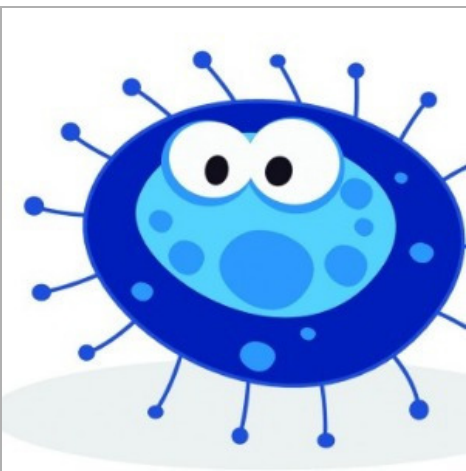

VERSION 1

\title{
(3) Use IPython to run bioconda tools in jupyter V.1
}

\author{
Alise J Ponsero ${ }^{1}$
}

${ }^{1}$ University of Arizona

Metagenomics class 2019

Alise J Ponsero

University of Arizona

\section{SEP 17, 2019}

\section{OPEN $\odot$ ACCESS}

DOI:

dx.doi.org/10.17504/protocol s.io.7ephjdn

Protocol Citation: Alise J Ponsero 2019. Use IPython to run bioconda tools in jupyter. protocols.io

https://dx.doi.org/10.17504/p rotocols. io. 7 ephjdn

License: This is an open access protocol distributed under the terms of the Creative Commons Attribution License, which permits unrestricted use, distribution, and reproduction in any medium, provided the original author and source are credited

Protocol status: Working We use this protocol and it's working

Created: Sep 17, 2019

Last Modified: Sep 17, 2019

PROTOCOL integer ID:

27823

Keywords: python, notebooks

\section{ABSTRACT}

Learn how to run shell commands directly in your jupyter notebook using IPython

\section{BEFORE START INSTRUCTIONS}

For this protocol, you'll need Anaconda/miniconda and jupyter installed on your machine. 
1 Meet Bioconda. Bioconda is a project aiming to integrate bioinformatic tools in the conda package management system. They have over 6000 bioinformatic tools available and a repository of recipe on how to use them.

Yes, Bioconda is your new best friend.

You can browse for your tool of interest in Bioconda through their tool repository. For this tutorial we will install and run Mothur.

\section{installing Mothur}

2 Open Anaconda Navigator and go to your environment manager. Select the class environment and search for the Mothur package in the "not installed" packages list.

Install Mothur in the environment. If you don't remember how to do this. Do not panic and go back to the Anaconda protocol (https://www.protocols.io/view/first-steps-using-a-jupyter-notebook6uphevn).

The installation will take some time, therefore you can read this very serious paper about Inuit poop knives.

2.1 For Miniconda users, open your terminal and type :

source activate class 2019

You should have your environment correctly loaded. Then type :

conda install Mothur

Confirm the installation by typing "y" and wait patiently for the installation.

\section{Using IPython}

3 Is Mothur is installed in your conda environment? Great! Now open a Python jupyter notebook in your Document folder. 
4 Think of IPython as a bridge between Python and shell commands. It's basically allowing you to run shell command lines directly into your Python Notebook! Are you excited? Ok, let's try it!

As you (should) know, in shell you can print a text using the "echo" command. Like this :

echo "I love metagenomics"

Well, let's say you REALLY want to use the "echo" command in your notebook, then IPython allows you to just type :

!echo "I love metagenomics"

Now, try it and run the cell ! You should see

In [10]: !echo "I love metagenomics"

I love metagenomics

In $[\quad]:$ |

And the same idea applies to Is, pwd, mkdir... all your favorite commands are there! All? Well, almost all...

5 If you play with IPython long enough, you'll quickly notice that !cd doesn't exactly work as it is supposed to. If you try to run

$$
\text { !cd . . }
$$

You won't see an error, but you won't have changed directory either... This is because each cell from the notebook is run as a temporary sub-shell. It opened a shell, ran the cd .. and then closed it. So you're back to square one.

To run these commands, you need to use a \% instead of the ! 
$\% \mathrm{~cd}$

Now, it should work! And even better, if you have the "automagic" IPython function installed, you don't need to use the "\%". You can simply run :

$\operatorname{cd} \ldots$

You have automagic functions for the main shell commands (Is, cd, cat, man, mkdir, mv, pwd, rm and rmdir).

\section{running Mothur}

6 So let's use IPython to run Mothur. To do so, you need to find where conda installs your packages. The path to this folder should look like this :

'/Users/[USERNAME]/anaconda3/envs/class_2019/bin'

Once you know where is you bin folder, you can run Mothur using the command (here showing the output of the help command in Mothur):

!/Users/[USERNAME]/anaconda3/envs/class_2019/bin/mothur "\#help( );"

Note that Mothur is by default an interactive tool. You cannot use this feature in a notebook, however, you can use Mothur command line mode. 